\title{
Abundance constraints and direct redshift measurement of the diffuse X-ray emission from a distant cluster of galaxies
}

\author{
Yasuhiro Hashimoto $^{1}$, X. Barcons ${ }^{2}$, H. Böhringer ${ }^{1}$, A. C. Fabian ${ }^{3}$, G. Hasinger ${ }^{1}$, V. Mainieri ${ }^{1}$, and H. Brunner ${ }^{1}$ \\ 1 Max-Planck-Institut für extraterrestrische Physik, Giessenbachstrasse, 85748 Garching, Germany \\ 2 Instituto de Física de Cantabraia (CSIC-UC), 39005 Santander, Spain \\ 3 Institute of Astronomy, Madingley Road, Cambridge, CB3 OHA, UK
}

Received 26 August 2003 / Accepted 12 January 2004

\begin{abstract}
We report on the XMM-Newton (XMM) observation of RX J1053.7+5735, one of the most distant X-ray selected clusters of galaxies, which also shows an unusual double-lobed X-ray morphology, indicative of a possible equal-mass cluster merger. The cluster was discovered during the ROSAT deep pointings in the direction of the Lockman Hole. All XMM Lockman Hole observations (PV, AO-1 \& AO-2 phases) with the European Photon Imaging Camera (EPIC) were combined for the analysis, totaling exposure times $\sim 648 \mathrm{ks}, 738 \mathrm{ks}$, and $758 \mathrm{ks}$ for pn, MOS1, and MOS2, respectively. With this "deep" dataset, we could detect the Fe $\mathrm{K}$ line and obtain a strong constraint on cluster metallicity, which is difficult to achieve for clusters at $z>1$. The best-fit abundance is $0.46_{-0.07}^{+0.11}$ times the solar value. The Fe line emission also allows us to directly estimate the redshift of diffuse gas, with a value $z=1.14_{-0.01}^{+0.01}$. This is one of the first clusters whose X-ray redshift is directly measured prior to the secure knowledge of cluster redshift by optical/NIR spectroscopy. We could also estimate the X-ray redshift separately for each of the two lobes in the double-lobed structure, and the result is consistent with the two lobes being part of one cluster system at the same redshift. Comparison with other metallicity measurements of nearby and distant clusters shows that there is little evolution in the ICM metallicity from $z \sim 1$ to the present.
\end{abstract}

Key words. X-rays: galaxies: clusters - cosmology: cosmological parameters - galaxies: high-redshift

\section{Introduction}

The heavy element abundances and their distribution in the intracluster medium (ICM) of galaxy clusters provides us with important information on the evolution of the cluster galaxies, the clusters themselves, and possibly the universe as a whole. By tracing the evolution of the global metal content of the ICM, one can obtain useful information on the epoch where the last major star formation in cluster galaxies took place and enriched the diffuse cluster gas. If clusters are fair samples of the universe, then studies of the evolution of the ICM metallicity also constrain the star formation history in the universe. Supernova explosions, which are the main contributor to metal enrichment, may also provide a significant source of heating for the ICM. Therefore, a knowledge of the metal content of galaxy clusters allows one to estimate the total amount of energy supplied by supernova explosions (e.g. Pipino et al. 2002).

$\mathrm{X}$-ray spectroscopic observations provide a powerful means to probe the metal content of the diffuse gas in clusters. In the late 70's, the iron line emission was discovered in the X-ray spectra of galaxy clusters (e.g. Serlemitsos et al. 1977). It was later found that the ICM in $z \sim 0$ clusters of

Send offprint requests to: Yasuhiro Hashimoto,

e-mail: hashimot@mpe.mpg.de galaxies has been enriched in iron to approximately $1 / 3$ of the solar value (e.g. Edge \& Stewart 1991). ASCA and BeppoSAX showed that the metallicity of the ICM stays constant at its local value $Z=0.3 Z_{\odot}$ out to $z \sim 0.4$ (e.g. Mushotzky \& Loewenstein 1997; Allen \& Fabian 1998; Della Ceca et al. 2000; Matsumoto et al. 2000; Ettori et al. 2001). These observations clearly indicate that a significant amount of the metals produced by supernovae were injected by $z \geq 0.4$.

With Chandra and XMM-Newton (XMM) observations on distant clusters, the evolution of chemical abundances beyond $z=0.4$ can be investigated, thus extending the previous analysis based on ASCA and BeppoSAX data (e.g. Jeltema et al. 2001; Arnaud et al. 2002; Holden et al. 2002; Jones et al. 2003; Maughan et al. 2003; Worrall et al. 2003; Rosati et al. 2004). Tozzi et al. (2003) analyzed mostly-Chandra data of 18 distant clusters with redshift $0.3<z<1.3$. They report, using a combined spectral fit of cluster subsamples in different redshift bins, that the mean Fe abundance at $z \sim 0.8$ is $Z=0.25_{-0.06}^{+0.04} Z_{\odot}$, consistent with the local metallicity value. Unfortunately, for clusters at redshift $z>1$, they could only provide weak constraints for the metallicity $\left(Z=0.21_{-0.05}^{+0.12} Z_{\odot}\right)$ even after combining four clusters.

The cluster RX J1053.7+5735, which shows an unusual double-lobed X-ray morphology, was discovered during our 
deep (1.31 Ms) ROSAT HRI pointings (Hasinger et al. 1998a), in the direction of the "Lockman Hole", a line of sight with exceptionally low HI column density. The angular size of the source is $1.7 \times 0.7 \operatorname{arcmin}^{2}$ and two lobes are approximately 1 arcmin apart. The total X-ray flux of the entire source in the $0.5-2.0 \mathrm{keV}$ band is $2 \times 10^{-14} \mathrm{erg} \mathrm{cm}^{-2} \mathrm{~s}^{-1}$ (Hasinger et al. 1998b). Our subsequent deep optical/NIR imaging follow-ups $(V<26.5, R<25, I<25, K<20.5)$ with LRIS and NIRC on Keck, and the Calar Alto Omega Prime camera revealed a bright 7 arcsec-long arc with an integrated magnitude of $R=21.4$ as well as an overdensity of galaxies in both X-ray lobes (e.g. Thompson et al. 2001). Further Keck LRIS/NIRSPEC spectroscopic observations on the bright arc and one of the brightest galaxies near the arc showed that the former is a lensed galaxy at a redshift $z=2.57$ while the latter is at a redshift of $z=1.263$ (Thompson et al. 2001). Deep VRIzJHK photometry data produced concordant photometric redshifts for more than 10 objects at redshift of $z \sim 1.1-1.3$, confirming that at least the eastern lobe is a massive cluster at high redshift. The improbability of chance alignment and similarity of colors for the galaxies in the two $\mathrm{X}$-ray lobes were consistent with the western lobe also being at $z \sim 1.1-1.3$ (Thompson et al. 2001). Meanwhile, the X-ray data of the XMM observation (with a total effective exposure time $\sim 100 \mathrm{ks}$ ) performed during the PV phase for this cluster were analyzed (Hashimoto et al. 2002), yielding a best-fit temperature of $4.9_{-0.9}^{+1.5} \mathrm{keV}$, while the metallicity was poorly constrained with an upper limit on the iron abundance of $0.62 Z_{\odot}$.

Here we report on a deep $\sim 1 \mathrm{Ms}$ XMM observation of the cluster RX J1053.7+5735, based on the data obtained during the PV, AO-1, \& AO-2 phases. The paper is organized as follows. In Sect. 2, we briefly describe the observation and data reduction. In Sect. 3, we first present spectroscopic analysis of the entire cluster, then of each lobe. In Sect. 4, we present a discussion of the metallicity evolution, as well as the comparison between the X-ray redshift and optical/infrared redshifts. All uncertainties quoted are at the $68 \%$ confidence levels for a one interesting parameter fit. Throughout the paper, we use $H_{0}=65 \mathrm{~km} \mathrm{~s}^{-1} \mathrm{Mpc}^{-1}, \Omega_{m}=0.3$, and $\Omega_{\Lambda}=0.7$

\section{Observations and data reduction}

The cluster RX J1053.7+5735 was observed as a part of the XMM Lockman Hole observation with European Photon Imaging Camera (EPIC) during the period April 27 May 19, 2000 (rev. 70, 71, 73, 74, and 81) for $\sim 170 \mathrm{ks}$ (PV phase), the period Oct. 25 - Nov. 4, 2001 (rev. 344, 345, and 349) for $\sim 199 \mathrm{ks}$ (AO-1), and the period Oct. $15-$ Dec. 7, 2002 (rev. 522-528, and 544-548) for $\sim 920 \mathrm{ks}$ (AO-2). Both EPIC cameras, pn and MOS, were operated in Full Frame mode and with the medium optical blocking filter for the bulk of time, except for the PV observation, where the thin filter was used for pn, and thin and thick filters were used for MOS. The light curves were visually inspected, and we discarded the data corresponding to the high particle background $(>8 \mathrm{ct} / \mathrm{s}$ for the pn, and $>5.2 \mathrm{ct} / \mathrm{s}$ for MOS1 and MOS2 inside the entire fov in the $0.5-10 \mathrm{keV}$ band). The data with pattern $>4$ and pattern $>12$ were also excluded for pn and MOS, respectively,

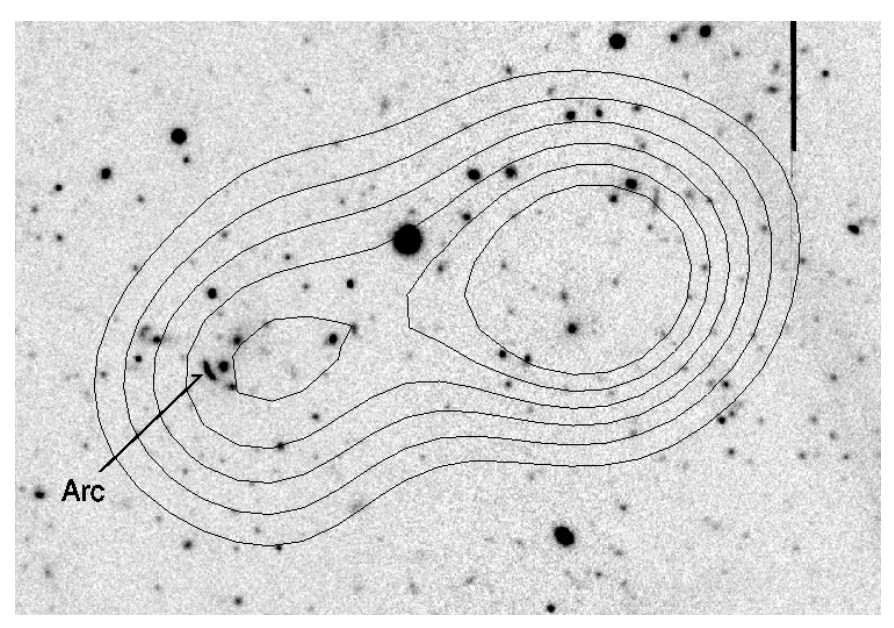

Fig. 1. The contours of XMM image of the cluster RX J1053.7+5735 overlaid on a CFHT $I$ band image. The image was created by combining all events in the $0.2-8 \mathrm{keV}$ band from three (pn, MOS1, \& MOS2) cameras. North is up and East is left. The image is $2.3 \times 1.5$ on a side. The raw data were smoothed with a Gaussian with $\sigma=7^{\prime \prime}$. The lowest contour is 1.9 counts $\operatorname{arcsec}^{-2}$ and the contour interval is 0.2 counts $\operatorname{arcsec}^{-2}$.

retaining only single and double events for pn, and single to quadruple events for MOS. We further removed those events with low spectra quality (i.e. FLAG $>0$ ). The remaining exposure times after this cleaning is approximately $648 \mathrm{ks}$, $738 \mathrm{ks}$ and $758 \mathrm{ks}$ for pn, MOS1, and MOS2, respectively.

\section{Results}

\subsection{Overall cluster analysis}

The spectra were extracted from an elliptical region centered at the cluster image with a semi-major/minor axis 1.2 and $0{ }^{\prime} 6$, respectively The backgrounds were estimated from a co-centric annulus region surrounding the cluster with 1'3 (inner) and 2'.1 (outer) radii, after removing point sources. We obtain 4605 and 4305 net source counts (in the $0.2-10.0 \mathrm{keV}$ band) for pn and $\operatorname{MOS}(1+2)$, respectively.

To correct for vignetting effects, we used the SAS V5.4.1 task EVIGWEIGHT (Arnaud et al. 2002), so that we can use on-axis effective areas. This task computes a weight coefficient for each event, defined as the ratio of the effective area at the photon position and energy to the central effective area at that energy, including filter transmission and quantum efficiency. Redistribution matrices are generated by the SAS RMFGEN for each pointing, filter, and detector, and then combined for joint analysis. We regrouped all the source spectra so that no energy bin had fewer than 50 counts. We fitted the spectra with a redshifted MEKAL model (e.g. Mewe et al. 1985; Kaastra \& Mewe 1993) using the XSPEC V11.0 (Arnaud 1996). We fit pn and MOS data (MOS1 and MOS2 are merged) jointly with common temperature, abundance, and redshift, while using separate normalization for each dataset, and fixing the column density of neutral hydrogen $N_{\mathrm{H}}$ at a value of $5.6 \times 10^{19} \mathrm{~cm}^{-2}$ (Dickey \& Lockman 1990).

In Fig. 2, the results of the best-fits are shown, while Fig. 3 shows the two-parameter $\chi^{2}$ contours for the cluster metallicity 
data and folded model

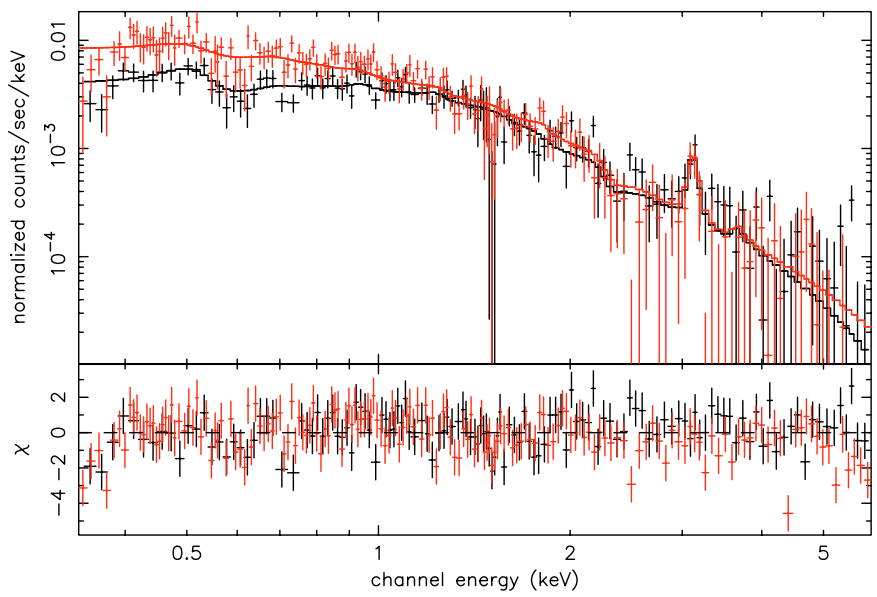

Fig. 2. Rebinned spectra, residuals, and best-fit models for cluster RX J1053.7+5735 with MOS1+2 (lower spectrum), and pn (upper spectrum) cameras. The crosses are the observed spectra and the solid lines denote best-fit models.

and X-ray temperature. The best-fit values based on a simultaneous fit are $k T=3.9_{-0.2}^{+0.2} \mathrm{keV}$, an abundance of $0.46_{-0.07}^{+0.11}$ times the solar value, and a redshift $z=1.14_{-0.01}^{+0.01}$. Using the bestfit model parameters, we derived an unabsorbed (0.2-10) keV flux of $f_{0.2-10}=2.8 \times 10^{-14} \mathrm{erg} \mathrm{cm}^{-2} \mathrm{~s}^{-1}$, corresponding to a luminosity in the cluster rest frame of $L_{0.2-10}=2.4 \times 10^{44} \mathrm{erg} \mathrm{s}^{-1}$ and a bolometric luminosity of $L_{\mathrm{bol}}=2.8 \times 10^{44} \mathrm{erg} \mathrm{s}^{-1}$. Note that the best-fit redshift is consistent with the redshift range derived by the photometric redshift technique (Thompson et al. 2001), while it is significantly lower than the NIR spectroscopic redshift $(z=1.26)$ of one galaxy in the eastern lobe near the gravitational arc. We will further comment about this point in Sect. 4.

\subsection{Substructure}

As illustrated in Fig. 1, the cluster RX J1053.7+5735 shows a clearly double-lobed structure, which extends roughly in the east-west direction. Using a circular (radius $=0.47$ ) extraction region centered at each lobe, X-ray characteristics are investigated for the substructure.

Figure 4 shows X-ray spectra and residuals for the eastern lobe (top panel) and for the western lobe (bottom panel). Figure 5 shows two-dimensional $\chi^{2}$ contours at $68.3 \%, 90 \%$, and $99 \%$ confidence levels $\left(\Delta \chi^{2}=2.30,4.61\right.$ and 9.21) for the eastern and western lobes of the cluster temperature and redshift, obtained from the joint fit to the pn and MOS data. Our fitting for each lobe shows that $k T$ is $3.4_{-0.1}^{+0.2} \mathrm{keV}$ and $4.4_{-0.3}^{+0.3} \mathrm{keV}$, the redshift is $1.16_{-0.03}^{+0.02}$ and $1.14_{-0.01}^{+0.02}$, and the abundance is $0.41_{-0.16}^{+0.18}$ and $0.62_{-0.14}^{+0.15}$ for eastern and western lobe, respectively (Table 1). Note that the redshifts of two lobes agree within a statistical limit, consistent with the interpretation that two lobes are at close physical distance.

To investigate the indication of detailed temperature variations in the cluster, we created an X-ray "hardness ratio" map,

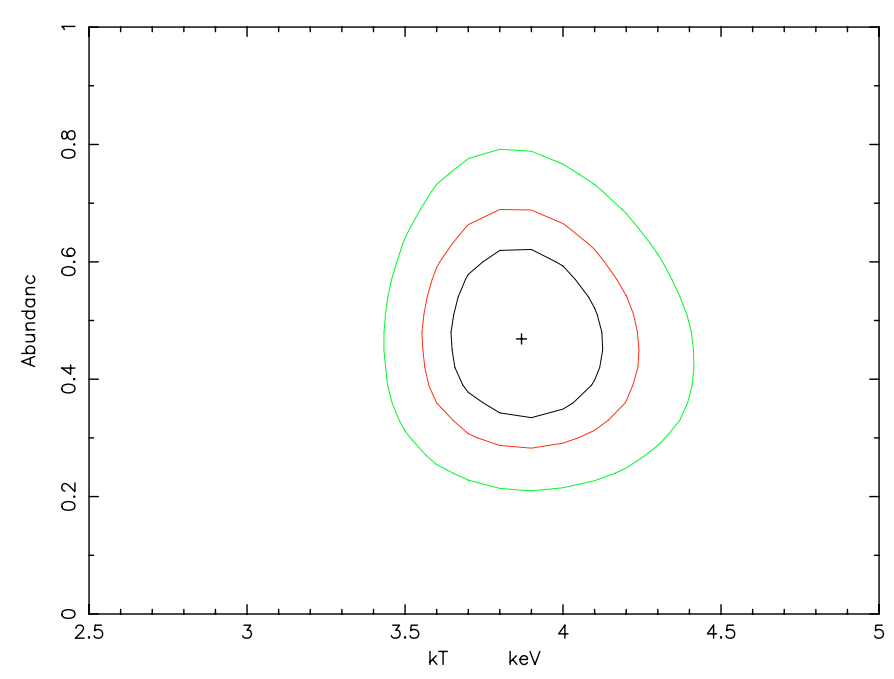

Fig. 3. Two-dimensional $\chi^{2}$ contours at $68.3 \%$, 90\%, and $99 \%$ confidence levels $\left(\Delta \chi^{2}=2.30,4.61\right.$ and 9.21) for the temperature $k T$ and the abundance $Z / Z_{\odot}$ of the cluster RX J1053.7+5735. Both the temperature and the abundance are obtained from the joint fit to the pn and MOS data.

whose structure may be interpreted as temperature variations. The X-ray hardness ratio map is created, first, by making hard $(1-8 \mathrm{keV})$ and soft $(0.2-1 \mathrm{keV})$ band images, then by dividing the hard band image by the soft band image. Two images are separately smoothed before the division with a Gaussian with $\sigma=7^{\prime \prime}$. This map is shown in Fig. 6. The full-band $(0.2-8 \mathrm{keV})$ contours are overlaid on the hardness map. Inset shows the hardness ratio map of the $\beta$ model $(\beta=0.67$, $r_{\mathrm{c}}=17^{\prime \prime} .2$ ) convolved with the detector PSF at the position of the cluster (westlobe). The inset covers the region of the sky identical to the main figure of Fig. 6 . The profile of each pointing and each detector in $0.2-1,1-2,2-5$, and $5-8 \mathrm{keV}$ bands are separately created by the SAS V.6.0 emldetect task with a parameter extentmodel $=$ beta. The hard band $(1-8 \mathrm{kev})$ is split into three bands and later combined, in order to reflect the cluster X-ray flux in each band. The scale bar in the inset is shown as a reference for the image-size (the color-scales are the same as the main figure). The inset demonstrates that the energy dependence of the detector PSF has negligible effect on the cluster hardness ratio map, where the bulk of the cluster emission is below $5 \mathrm{keV}$. Figure 6 shows that the coldest part of the western lobe may be slightly off-centered toward west, while the temperature structures of the eastern lobe is aligned with respect to the full-band contours. The region between the two lobes seems to have similar temperature with the outer region of each lobe, and seems hotter than the bulk of the cluster temperature, however, the influence from the cluster-cluster collision is unclear based on our data. We will further discuss about this point in Sect. 4. 

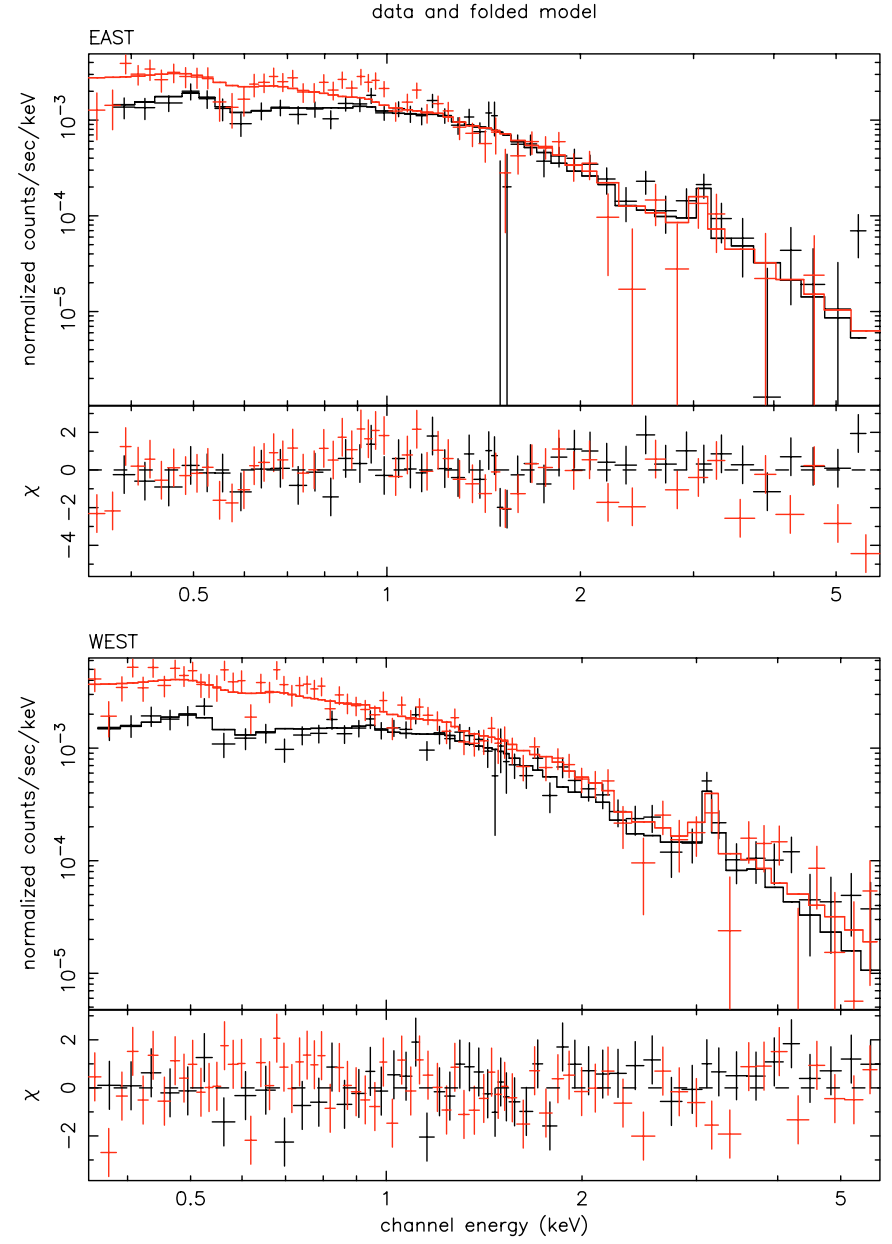

Fig. 4. X-ray spectrum and residuals for the east lobe (top panel), and for the west lobe (bottom panel).

Table 1. Comparison between the two lobes (pn+MOS1+MOS2).

\begin{tabular}{lrrrl}
\hline \hline Lobe & $k T$ & $Z / Z_{\odot}$ & Redshift & R. $\chi^{2}$ (d.o.f.) \\
& $(\mathrm{keV})$ & & $(z)$ & \\
\hline East & $3.4_{-0.1}^{+0.2}$ & $0.41_{-0.16}^{+0.18}$ & $1.16_{-0.03}^{+0.02}$ & $1.47(99)$ \\
West & $4.4_{-0.3}^{+0.3}$ & $0.62_{-0.14}^{+0.15}$ & $1.14_{-0.01}^{+0.02}$ & $1.00(119)$ \\
ALL & $3.9_{-0.2}^{+0.2}$ & $0.46_{-0.07}^{+0.11}$ & $1.14_{-0.01}^{+0.01}$ & $1.17(301)$ \\
\hline
\end{tabular}

\section{Discussion}

\subsection{Cluster redshift}

The redshift of the cluster diffuse gas directly estimated by fitting the X-ray data is $z=1.14_{-0.01}^{+0.01}$. This value is within the cluster redshift-range obtained with the photometric redshift technique. However, it is significantly lower than a redshift $(z=1.26)$, of one cD-like galaxy near the eastern-lobe arc, obtained by near infrared spectroscopy. The infrared redshift is practically defined by one line detected at $1.485 \mu \mathrm{m}$, which Thompson et al. (2001) interpreted as $\mathrm{H} \alpha$ in light of its photometric redshift.

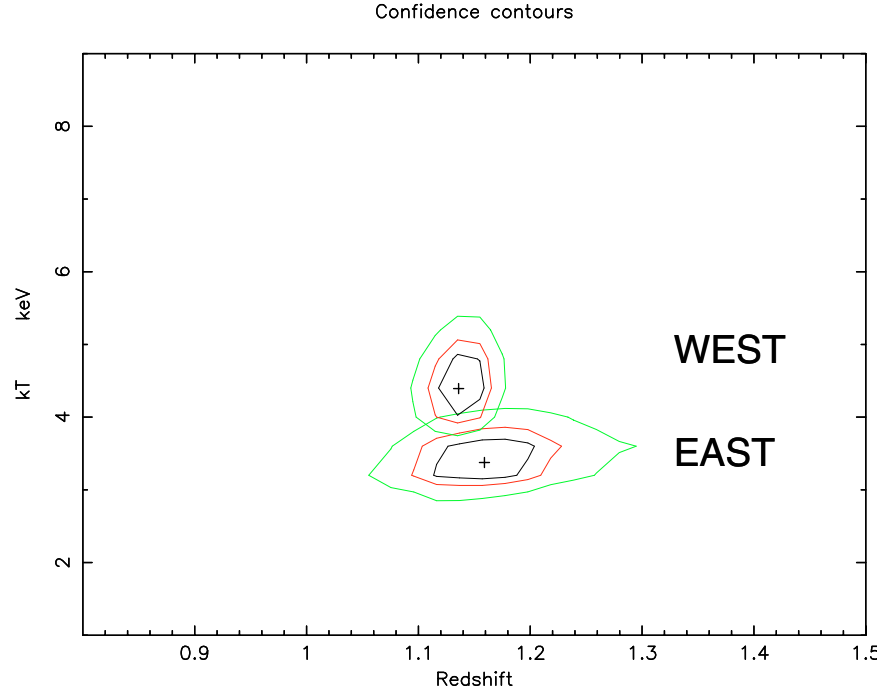

Fig. 5. Two-dimensional $\chi^{2}$ contours at $68.3 \%, 90 \%$, and $99 \%$ confidence levels $\left(\Delta \chi^{2}=2.30,4.61\right.$ and 9.21) for the temperature $k T$ and the redshift of the eastern and western lobes of cluster RX J1053.7+5735.

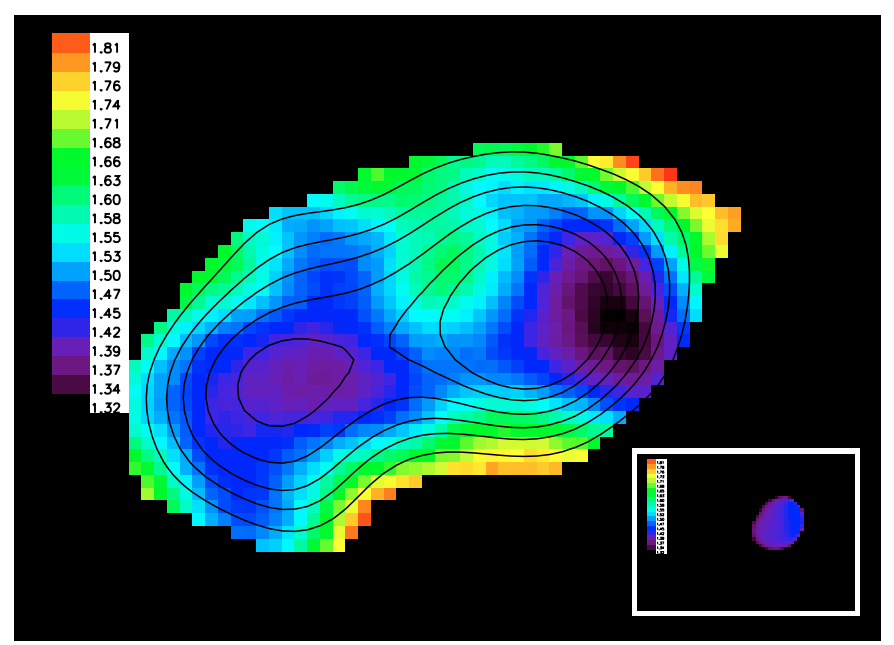

Fig. 6. The X-ray hardness ratio map where the hard band image $(1-8 \mathrm{keV})$ is divided by soft band image $(0.2-1 \mathrm{keV})$. Both images are separately smoothed, before the division, with a Gaussian with $\sigma=7^{\prime \prime}$. The full-band $(0.2-8 \mathrm{keV})$ contours are overlaid on the hardness ratio map. Illustratively, the ratio $=1.8$ here roughly corresponds to $k T \sim 9-10 \mathrm{keV}$, while the value 1.3 corresponds to $k T \sim 3-4 \mathrm{keV}$. Inset shows the hardness ratio map of the $\beta$ model convolved with the detector point spread function (PSF) at the position of the cluster (westlobe), demonstrating that the energy dependence of the detector PSF has negligible effect on the cluster hardness ratio map.

If the redshift of this $\mathrm{cD}$-like galaxy were at $z=1.14$, as indicated by the X-ray redshift, then this emission line detected in the $\mathrm{cD}$-like galaxy would not correspond to any prominent line. Moreover, no line is detected at $1.404 \mu \mathrm{m}$ where possible $\mathrm{H} \alpha$ line should be located, if the galaxy is indeed at $z=1.14$. Therefore, it is unlikely that redshift of this particular galaxy is at $z=1.14$ 
The simplest explanation for this redshift discrepancy is: the cD-like galaxy is in the background of the cluster, and therefore, not a member of the cluster (relative velocity $\sim 17000 \mathrm{~km} \mathrm{~s}^{-1}$ ). Even with the apparent proximity of the cD-like galaxy to the gravitational arc and to the center of the $\mathrm{X}$-ray contour, this explanation is indeed possible. In fact, there is another $\mathrm{cD}$ galaxy candidate equally close to the center of the X-ray contour. We obtained DEIMOS/Keck spectroscopy of this galaxy and our preliminary analysis shows that the redshift of the galaxy is consistent with that of X-ray diffuse gas.

\subsection{Dynamical state of the cluster}

The fact that the X-ray morphology of RX J1053.7+5735 is double-lobed suggests that we may be witnessing a possible equal-mass merger event at $z>1$. We could estimate the X-ray redshift separately for each lobe, and the result is consistent with the interpretation that two lobes are a part of one cluster system at the same redshift, although the dynamical state of the system cannot be definitively known from the redshiftinformation alone.

As for the Tx distribution, as shown in Fig. 6, the inter-lobe region seems hotter than the bulk of each lobe, however it is not clear from the figure that the temperature in that region is actually enhanced, by the mechanism such as a shock induced by the cluster "collision". Relatively abrupt temperature boundary between the low temperature region in the western lobe and the inter-lobe region may be similar with the "cold fronts" observed in local clusters (e.g. Markevitch et al. 2000; Vikhlinin et al. 2001). In these "cold fronts", a sharp temperature gradient forms when a cooler gas blob propagates in a hotter ambient gas. The cold western lobe spot which shows a steep temperature gradient relative to an intermediate hot cluster-intersection region might be interpreted as one such "cold front". Note, however, that our abrupt temperature boundary does not seem to match exactly the densest part of the gas (as shown by the contours) and therefore this tentative interpretation has to be taken with some caution.

Relatively high Fe abundance of RX J1053.7+5735 is somewhat surprising, particularly considering its redshift. However, it may be explained by the possible existence of "densed core" (or "colling flow") in the cluster, and its relation to the $\mathrm{Fe}$ abundance (e.g. Allen \& Fabian 1998; see also De Grandi \& Molendi 2001). The hardness ratio map of RX J1053.7+5735 may indicate that the temperature is cooler at the center of each lobe, which is consistent with the cooling flow interpretation. However, this "densed core" feature has been considered to be associated with a relaxed dynamical state of clusters (e.g. McGlynn \& Fabian 1984; Edge et al. 1992). If the two lobes of RX J1053.7+5735 are indeed interacting, and are in the process of nearly-equal-mass merger, it would be unlikely that this dynamical state exists inside the cluster, and therefore unlikely to have the "densed core" profile. Unfortunately, the spatial resolution of our data is not sufficient enough to directly investigate the core profile of the cluster RX J1053.7+5735, and therefore, the implied dynamical state of the cluster remains unknown. Future X-ray

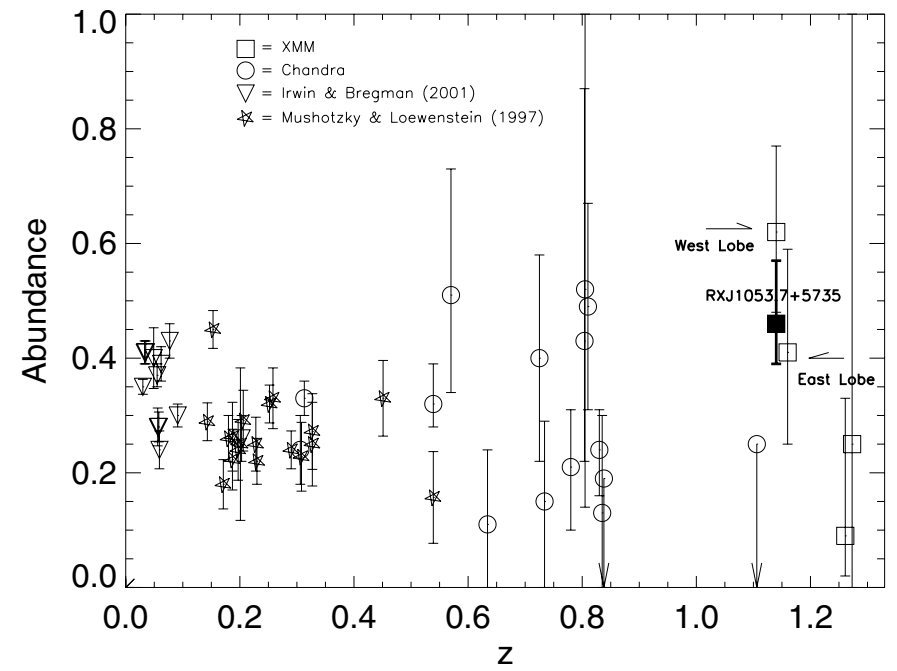

Fig. 7. New XMM abundance and its $1 \sigma$ error (68.3\%) for RX J1053.7+5735 plotted with high redshift clusters from Tozzi et al. (2003). For comparison, the low redshift samples from Mushotzky \& Loewenstein (1997), and Irwin \& Bregman (2001) are also plotted.

observations with high spatial resolution, as well as the optical/NIR spectroscopic observations of individual cluster members, are needed for more definitive statement about the dynamical state of the cluster.

\subsection{Abundance vs. redshift}

In Fig. 7, we compare our newly measured metallicity for RX J1053.7+5735 with other high redshift clusters observed by XMM or Chandra from Tozzi et al. (2003). For comparison, the low redshift samples from Mushotzky \& Loewenstein (1997), and Irwin \& Bregman (2001) are also plotted. The black square denotes the new abundance for RX J1053.7+5735. The eastern and western lobes of the cluster are also separately shown (labeled as "East Lobe" and "West Lobe"). The error bars on the abundance are one dimensional $68 \%$ confidence range. Some $90 \%$ errors quoted in the literature are converted to $68 \%$ error by multiplying by a factor $1 / 1.6$.

Several studies report a hint of a weak dependence of global Fe abundance on the temperature in such a way that lower temperature clusters tend to have larger $\mathrm{Fe}$ abundances than hot clusters (e.g. Yamashita 1992; Fabian et al. 1994; Mushotzky \& Loewenstein 1997; Matsumoto et al. 2000; Tozzi et al. 2003, see however Fukazawa et al. 1998; Fukazawa et al. 2000). This dependence may be related to the correlation between the core profile and $\mathrm{Fe}$ abundance, which indicates that more "densed core" or "cooling flow" clusters show the higher Fe abundance (e.g. Allen \& Fabian 1998). Regardless of the origin of this Fe dependence on the cluster temperature, Tozzi et al. (2003) subdivided their cluster sample according to the temperature (the "hot" cluster subsample with $k T>5 \mathrm{keV}$ and the "cold" cluster subsample with $k T<5 \mathrm{keV}$ ). They investigated the abundance vs. redshift relation separately for each subsample using a combined fit across all of the subsample clusters in different redshift bins, and reported that at least the high 


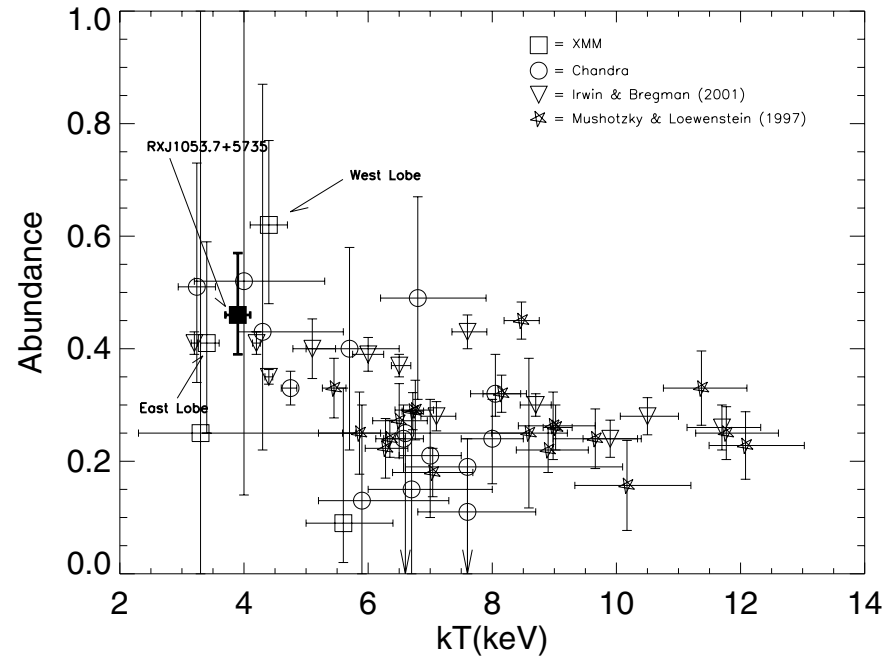

Fig. 8. New XMM abundance and Tx and their $1 \sigma$ errors $(68.3 \%)$ for RX J1053.7+5735 plotted with high redshift clusters from Tozzi et al. (2003). The low redshift samples from Mushotzky \& Loewenstein (1997), and Irwin \& Bregman (2001) are also plotted.

temperature subsample reveals no sign of the evolution in their average abundances up to $z \sim 1.2$.

Figure 8 shows the abundance vs. temperature relation for the same sample as Fig. 7. The symbols are identical with Fig. 7. The error bars on the temperature are again $68 \%$ confidence range. The cluster RX J1053.7+5735 roughly follows a weak trend that lower temperature clusters tend to have larger iron abundances than hot clusters. Figure 9 shows the abundance vs. temperature relation for the "hotter" clusters $(k T>5 \mathrm{keV}$ : Fig. 9a), and "cooler" clusters $(k T<5 \mathrm{keV}$ : Fig. 9b). Again, the black square denotes the new abundance for RX J1053.7+5735, with eastern and western lobes separately shown. For both subsamples, there is no statisticallysignificant redshift dependence, while the mean abundance is somewhat higher for the cooler subsample. The result is consistent with no-evolution of the Fe abundance at $z \sim 1.2$, supporting the scenario that much of the enrichment and star formation took place earlier.

The cluster RX J1053.7+5735 is now one of the first clusters whose X-ray redshift is directly measured prior to the secure knowledge of the cluster redshift obtained with optical/NIR spectroscopy. Deep pointings with the current X-ray satellites provide a powerful means to study the high-redshift clusters, enabling us to directly measure the redshifts, and to probe the metal contents, of the diffuse gas in the clusters. The high-throughput X-ray spectroscopic mission in the future, such as Con-X and XEUS, should make these studies almost a routine.

Acknowledgements. We thank Pat Henry for a careful reading of the paper and useful comments. XB acknowledges partial financial support by the Spanish Ministerio de Ciencia y Tecnología, under project AYA2000-1690. We acknowledge referee's comments which improved the manuscript.
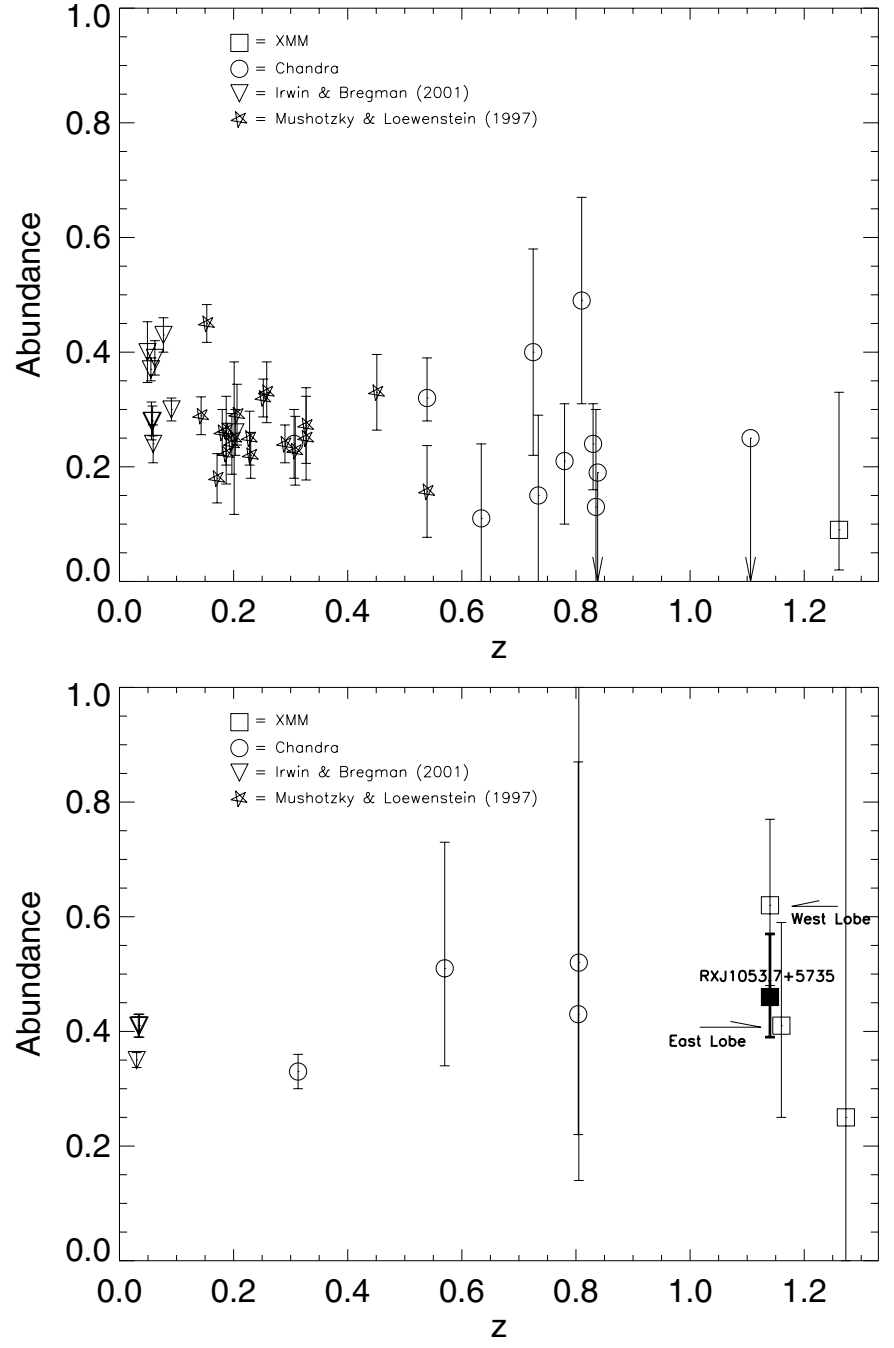

Fig. 9. Abundance vs. redshift for two subsets of clusters defined by X-ray temperature. Top panel: clusters with $k T>5 \mathrm{keV}$, Bottom panel: clusters with $k T<5 \mathrm{keV}$.

Note added in proof. For high resdshift clusters, the "effective" spatial resolution becomes lower because of the smaller angular size of the clusters. Some caution has to be exercised in interpreting the global X-ray characteritics of clusters, in particular high redshift ones, due to the possible contamination from unresolved point sources which may exist inside or in the direction of the clusters.

\section{References}

Allen, S. W., \& Fabian, A. C. 1998, MNRAS, 297, L63

Arnaud, K. A. 1996, ASP Conf. Ser., 101, 17

Arnaud, M., Majerowicz, S., Neumann, D. M., et al. 2002, A\&A, 390, 27

De Grandi, S., \& Molendi, S. 2001, ApJ, 551, 153

Della Ceca, R., Scaramella, R., Gioia, I. M., et al. 2000, A\&A, 353, 498

Dickey, J. M., \& Lockman, F. J. 1990, ARA\&A, 28, 215

Edge, A. C., \& Stewart, G. C. 1991, MNRAS, 252, 428

Edge, A. C., Stewart, G. C., \& Fabian, A. C. 1992, MNRAS, 255, 431

Ettori, S., Allen, S. W., \& Fabian, A. C. 2001, MNRAS, 322, 187 
Fabian, A. C., Crawford, C. S., Edge, A. C., \& Mushotzky, R. F. 1994, MNRAS, 267, 779

Fukazawa, Y., Makishima, K., Tamura, T, et al. 1998, PASJ, 50, 187

Fukazawa, Y., Makishima, K., Tamura, T., et al. 2000, MNRAS, 313, 21

Hashimoto, Y., Hasinger, G., Arnaud, M., Rosati, P., \& Miyaji, T. 2002, A\&A, 381, 841

Hasinger, G., Burg, R., Giacconi, R., et al. 1998a, A\&A, 329, 482

Hasinger, G., Giacconi, R., Gunn, J. E., et al. 1998b, A\&A, 340, L27

Holden, B. P., Stanford, S. A., Squires, G. K., et al. 2002, AJ, 124, 33

Irwin, J. A., \& Bregman, J. N. 2001, ApJ, 546, 150

Jeltema, T. E., Canizares, C. R., Bautz, M. W., et al. 2001, ApJ, 562, 124

Jones, C., Maughan, B. J., Ebeling, H., et al. 2003 [astro-ph/0304264]

Kaastra, J. S., \& Mewe, R. 1993, A\&AS, 97, 443

Matsumoto, H., Tsuru, T., Fukazawa, Y., et al. 2000, PASJ, 52, 153

Maughan, B. J., Jones, L. R., Ebeling, H., et al. 2003, ApJ, 587, 589
Mewe, R., Gronenschild, E. H. B. M., \& van den Oord, G. H. J. 1985, A\&AS, 62, 197

Markevitch, M., Ponman, T. J., Nulsen, P. E. J., et al. 2000, ApJ, 541, 542

McGlynn, T. A., \& Fabian, A. C. 1984, MNRAS, 208, 709

Mushotzky, R., \& Loewenstein, M. 1997, ApJ, 481, L63

Pipino, A., Matteucci, F., Borgani, S., et al. 2002, NewA, 7, 227

Rosati, P., Tozzi, P., Ettori, S., Mainieri, V., et al. 2004, AJ, in press [astro-ph/0309546]

Serlemitsos, P. J., Smith, B. W., Boldt, E. A., Holt, S. S., \& Swank, J. H. 1977, ApJ, 211, L63

Thompson, D., Pozzetti, L., Hasinger, G., et al. 2001, A\&A, 377, 778

Tozzi, P., Rosati, P., Ettori, S., et al. 2003, ApJ, 593, 705

Vikhlinin, A., Markevitch, M., \& Murray, S. S. 2001, ApJ, 551, 160

Worrall, D. M., \& Birkinshaw, M. 2003, MNRAS, 340, 1261

Yamashita, K. 1992, in Frontiers of X-ray Astronomy, ed. Y. Tanaka, \& K. Koyama (Tokyo: Universal Academy Press) 